\title{
Distributed Simulation of Amorphous Hydrogenated Silicon Films: Numerical Experiments on a Linux Based Computing Environment
}

\author{
Y.E. Gorbachev, M.A. Zatevakhin, V.V. Krzhizhanovskaya, A.A. Ignatiev, \\ V.Kh. Protopopov, N.V. Sokolova, and A.B. Witenberg \\ Institute for High Performance Computing and Data Bases \\ Fontanka 118, off. 55, St. Petersburg 198005, Russia \\ Gorbachev@hm.csa.ru] \\ http: //www.csa.ru/comphyga/PECVD
}

\begin{abstract}
Numerical scheme, grid generation and parallelization algorithm are presented for numerical simulation of complex process of silicon-based films growth in plasma enhanced chemical vapor deposition reactors. MPI-based computing environment and advanced interactive software with graphic user interface, real-time visualization system and Web access were recently developed to provide distributed parallel multitask calculation and visualization on Linux clusters. Analysis of system performance and cluster load balance showed the bottlenecks of parallel implementation and the ways of algorithms improvement.
\end{abstract}

\section{Introduction}

Numerical simulation of deposition process of amorphous hydrogenated silicon $(\alpha-\mathrm{Si}: \mathrm{H})$ films, the widespread material in modern microelectronics, is an important problem that has been solved over two recent decades. One of the most popular technologies of silicon-based films production is plasma enhanced chemical vapor deposition (PECVD), that has been studied for many years, so the knowledge about physics and chemistry of this phenomenon is rather rich in details. Though dozens of models were developed, describing the system with different levels of abstraction and accuracy, even the most advanced models still can not take into account all the numerous chemical kinetics, plasma physics and transport processes occur in industrial PECVD reactors due to terrific amount of computational time needed for thorough numerical simulation of the problem.

In that way, to find a balance between phenomenon knowledge and computational resources available, not only new physical models are developed, but also new rational numerical algorithms and computational environment are created, adjusted to modern computing facilities.

The project described in this paper is aimed to the development of efficient general purpose computer environment for large parameter space exploration of processes under consideration and to creation of high-performance software for end users working in chemical industry and for scientists studying PECVD processes. 
Although high performance is the vital factor for simulation of complex-shaped essentially 3D plasma deposition reactors, especially if it should be a short-time forecasting calculations for prediction and controlling of industrial processes, we oriented not to the unique most powerful supercomputers, but to the widely used Linux clusters, accumulated many virtues of parallel computers, and being the cheapest facilities on the factor of FLOPS cost, that is quite important parameter for scientific community. Nowadays, the maturity of clustering software, open sources and easiness of clusters assembling make them more and more popular in highperformance computing.

\section{Model Description}

For numerical experiments with cluster computing environment recently developed, $2 \mathrm{D}$ approach was chosen for modeling of $\alpha-\mathrm{Si}: \mathrm{H}$ films deposition from radiofrequency discharge silane plasma. We used the most reliable and well-studied method of computational fluid dynamics for simulation of the physical-chemical processes occur in PECVD reactors, based upon full Navier-Stockes equations. Under the conditions considered (characterized by low Mach and Reynolds numbers), for unsteady laminar flow of viscous compressible chemically active $N$-component gas mixture, these equations in conservation law 2D-form for a Cartesian coordinate system can be written as [1]:

$$
\frac{\partial}{\partial t} \int_{V} U d V+\oint_{S}(F+G) \cdot d S=\int_{V} H d V
$$

where $t$ is time and $U=\left(\rho, \rho u_{x}, \rho u_{y}, \rho e, \rho f_{1}, \ldots, \rho f_{N-l}\right)^{T}$ is a vector of conservative variables (here $\rho$ is density, $u_{x}, u_{y}$ are velocity components, $e$ is a total energy per mass unit, $f_{i}$ is mass fraction of $i$-species), $V$ is arbitrary gas volume and $d S$ is outward vector, normal to the element of surface $S$ which encloses volume $V$. Flux vectors $F$ and $G$ are associated with convective and viscous transfer correspondingly. Source term $H$ describes species production due to chemical reactions.

For calculation of the electron density, the hydrodynamic model of RF discharge between two planar parallel electrodes was used [2]. The electron and ion continuity equations were solved consistently with the Poisson equation for the electric field distribution.

Boundary conditions taking into account slipping, sticking and deposition effects for velocity, temperature and chemical components concentrations on the wall were applied [3,4].

\section{Numerical Scheme and Grid Generation}

Physical processes factorization method [5] was used for solving equations. According to this method the problem was split into inviscid and viscous "steps". At the first one, implicit ENO-scheme [6] was used in combination with bidiagonal 
algorithm [7] for implicit increment calculation, and for solving the "viscous" part, a new implicit finite-deference scheme was developed on the basis of [8].

Since an ordinary PECVD reactor has a complex shape (for example, one of the simplest reactors is shown on the figure 7), the multi-block grid generation algorithm was used, when whole computational domain is divided into simple blocks, and then in each block the regular grid is generated. The original method of mechanical analogy with deformed body [9] was applied for generation of non-uniform grids in non-rectangular blocks. The idea of this method is based upon solving equations of elastic body deformation theory:

Let us consider the rectangular plate $\mathrm{ABCD}$ made of elastic material with rectangular non-uniform grid plotted on it. Deformation of this plate, leading to the desirable shape of the reactor block, will accordingly deform the grid lines, thus creating the desired curvilinear grid. Figure 1 illustrates this approach. Grid points coordinates in this case are calculated from the elliptic equations of the theory of elasticity [9]. An example of multi-block grid for a complex geometry reactor is shown at the figure 2 .
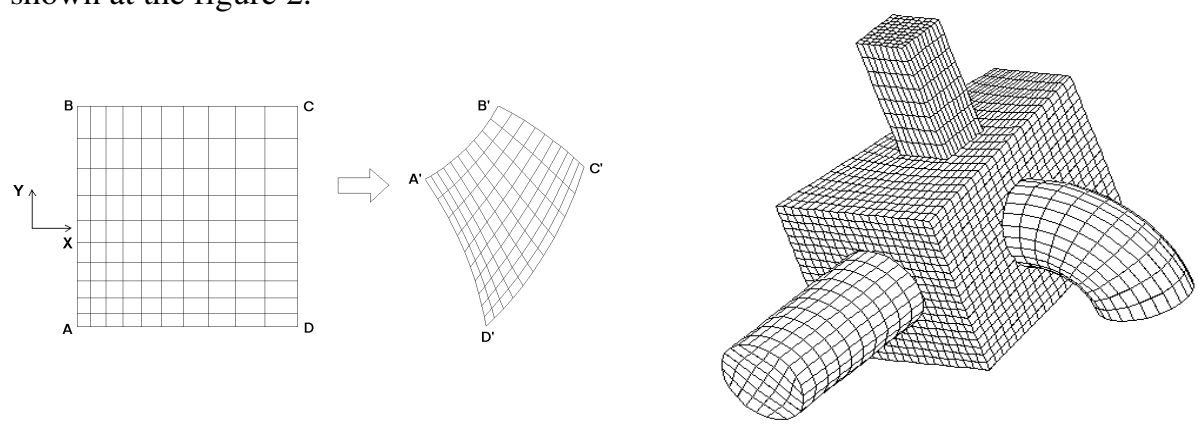

Fig. 1. Plate deformation method for grid generation

Fig. 2. Example of multi-block grid

\section{Parallelization}

Working out the effective computing environment for parallel computing, different levels of parallelization are considered. Because of the large number of parameters which influence upon film deposition rate and properties (pressure, gas and substrate temperatures, flow velocity, reactor volume and configuration, mixture composition and numerous plasma characteristics), for thorough investigation of the system behavior, many tasks with different initial conditions can be run in parallel on different processors, providing thus exploration of a large parameter space. This way of job-level parallelization is the most natural and efficient for many computational fluid dynamic problems which require significant efforts for parallelization of individual program algorithm and code. To utilize the advantages of this approach, a task Manager environment was developed, looking after cluster processors idleness and loading them with new jobs. When one of the processors finished its job, the Manager initiates a process with new initial data set, running over the values of parameters studied. 
However, this project is aimed to multipurpose multiscale simulation systems, including 3D simulation and visualization of complex-shaped industrial reactors, that implies terrifically heavy calculations, so parallelization on a task level is also needed. The algorithm and its implementation will be described in the next section.

The last and the most difficult problem of parallelizing and load balancing is the case when all the processors of computing cluster are already loaded with one or few tasks, and a user with the same priority (tasks with the lower priority are simply queue up) starts one or few more program(s). This problem recently arose when the computational system was tested by remote users working with the server cluster through the web-interface. The preliminary results of studying this problem are described in the Results section.

\section{Job-Level Parallelization Algorithm}

The natural approach to solve unsteady computational fluid dynamic problem with parallel computers is based on the following two obvious steps:

- Decomposition of computational domain into approximately equal sub-domains so that their number is equal to number of processors.

- At each time iteration each processor calculates only own sub-domain and then sends its results (decrements of variables) to the master processor (with rank 0 ).

The efficacy of this approach essentially depends on few factors:

- Simplicity of domain geometry, which permits to decompose whole domain into approximately equal sub-domains;

- Spatial uniformity of physical parameters fields and equality of total amount of operations to be done in every sub-domain;

- The ratio of data exchange time between two processes to calculation time for one processor.

Typical domain of PECVD reactor is usually rather complex, so the trivial geometric decomposition makes sub-domains unbalanced in number of grid points and total calculations to be done. A specific decomposition algorithm was developed in order to alleviate this imbalance. It is based upon multi-block regular grid with beam distribution for parallelization.

According to this concept the whole calculation domain is divided into number of primitive blocks which are mere boxes in $2 \mathrm{D}$ index space $\mathrm{i}$ and $\mathrm{j}$. For example, figure 3 shows a complex domain consisting of nine blocks. Each block is divided into 4-face cells so that in the whole domain has two families of beams: beams in "i" index direction and beams in " $\mathrm{j}$ " index direction. Each cell belongs to only one beam of each family and each beam includes a sequence of cells with adjacent faces so that one beam begins and finishes at the physical boundary (fig. 3 ). 


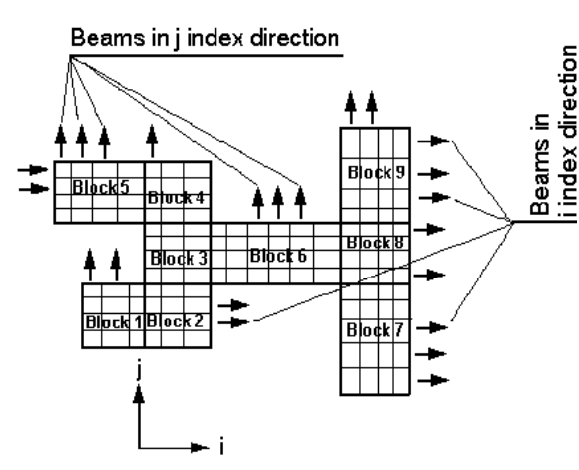

Fig. 3. Nine block domain

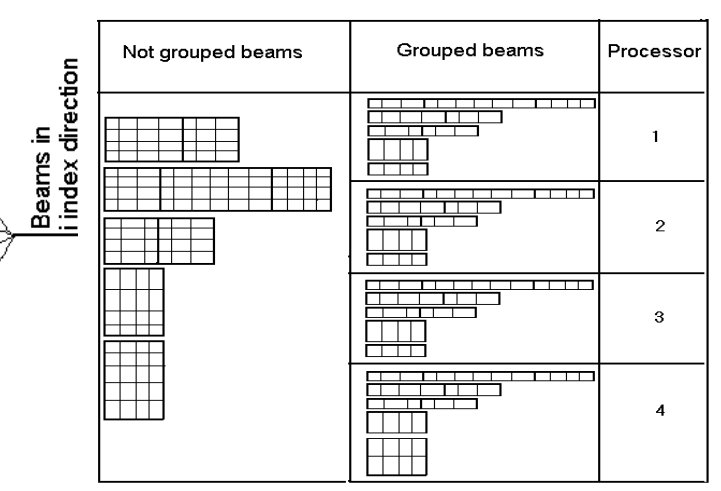

Fig. 4. Beam grouping inside "i" list

This beams decomposition has one important advantage for parallelization. This approach allows using uniform implicit scheme by applying sweep-type algorithms to the single beam and then to distribute beams among the processors.

Thus, the parallel algorithm of calculation at one time iteration boils down to the following steps:

- Formation of two lists of beams in two index directions ("i" list and "j" list) for the given domain.

- Compilation of NP groups of beams inside each of two lists. (NP - number of processors), so that each group has approximately equal number of cells.

From this point of view the best parallelization efficiency is achieved when the number of cells in every index direction of each block is divisible by $N P$. Otherwise beams are rearranged to meet the condition of approximate equality of number of the cells per every block, but the load balance will not be ideal because of some additional operations on the physical boundaries.

Figure 4 illustrates group distribution of beams for "i" list of our nine-blocks domain from fig. 3 for four processors. One can see that in this case loading of all processors is approximately equal. Similar grouping is made for " $\mathrm{j}$ " list.

- Based on this beam grouping, the work instructions are formed for each processor, listing the beams to be calculated by every processor.

- In the beginning of each time iteration master processor (with rank $=0$ ) sends all necessary information (conservative variables at n-time level) to each slave processor (with rank $>0$ ) according to its instructions. Each slave processor receives the data and computes the beams listed in its instructions.

- After performing its management duties, master processor fulfills its own calculation job and then waits for results (decrements of conservative variables)

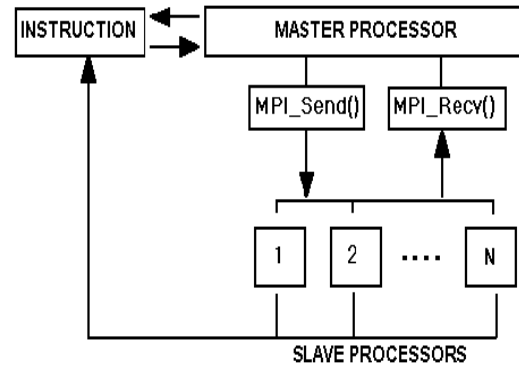

Fig. 5. Parallel algorithm 
from the slave processors. On receiving these results the Manager merges all data arrays into one decrements filed and repeats the previous step.

Briefly this algorithm for 1 time iteration is illustrated on the figure 5 .

\section{Implementation}

For implementation of numerical scheme and parallelization algorithm, MPI message passing interface was used in combination with computational $\mathrm{C}++$ core. To create a high-end software, an advanced graphic user interface was designed. For its realization we used $\mathrm{C} / \mathrm{C}++$ programming languages, widespread platform independent GTK+ graphic library and free Glade programming system. The application developed provides users with friendly intuitive interface that allows to control computing process carried out on a server cluster, to visualize numerical results in real-time (or postponed) mode, and to access chemical components data base or results archive. All programs of the software package are platform independent, and were compiled for OS Linux Red Hat 6.2.

To provide access to computational server for users studying PECVD process, the interface of remote user was developed. One of the prevalent technologies used for remote access to distributed computational resources is based upon Web browser as a client application. For this interface, we created HTML pages, Java applets, JavaScripts and CGI scripts. This system, like a local GUI, provides control over calculations and graphical presentation of current physical parameters fields, that is demonstrated on the figure 6 .

We have tested the computing environment on two Linux clusters. One consists of four two-processors nodes (each is Intel P-II $450 \mathrm{MHz}$ with $512 \mathrm{Mb}$ RAM) connected with Myrinet network adapter, and the second is 32-processors cluster of Intel P-III $600 \mathrm{MHz}$ with $512 \mathrm{Mb}$ RAM and SCI network communication.

After in-deep analysis of existing API interfaces for MPI library to the Myrinet communication environment, MPI-over-TCP was chosen, which allows to run many programs on cluster nodes simultaneously.

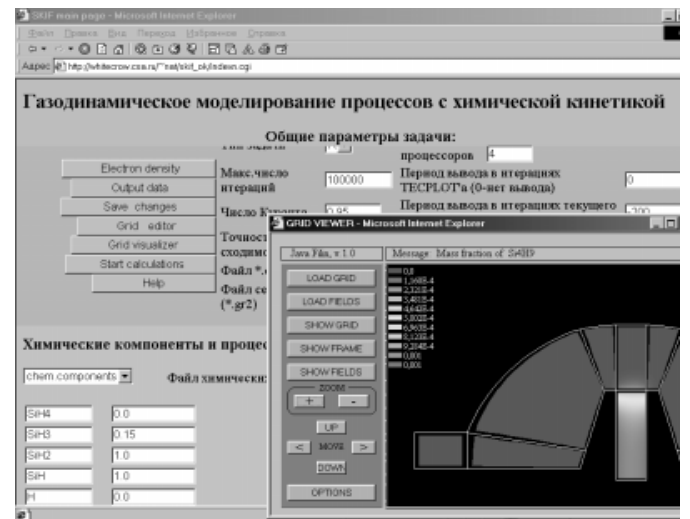

Fig. 6. Remote user interface

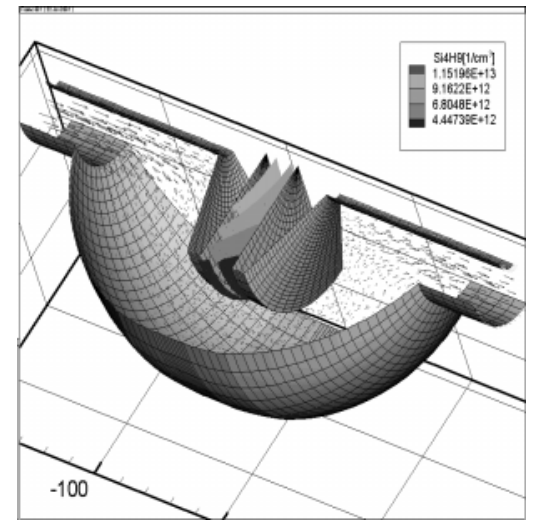

Fig. 7. Simulation results: $\mathrm{Si}_{4} \mathrm{H}_{9}$ formation 


\section{Results and Discussion}

A set of numerical experiments was carried out on a Linux-based computing environment, to verify the computational physical-chemical model and to evaluate the efficiency of parallelization algorithm. Reactor geometry, initial gas mixture and plasma discharge parameters were chosen corresponding to the real experimental data available [10]. Further details of the problem formulation can be found in [11].

Influence of different PECVD parameters on film growth and quality was studied, using the job-level parallelization. Running in exclusive mode, this problem did not cause imbalance of cluster processors load, thus a perfect efficiency was achieved with implementation of the task manager controlling processors idleness.

Few distinctive computational results are presented on figures 7 and 8 for the large-scale computational tasks. Here the influence of reactor geometry and pumping path is shown. In the first case (fig. 7, 8a), gas mixture was pumped into the reactor chamber through the inlets located outside discharge area, while in the second case (fig. 8b) mixture flows through a pump tube connected to one of the electrodes. Figure 7 shows the field of $\mathrm{Si}_{4} \mathrm{H}_{9}$ component (so called higher silane) concentration, and figures $8 \mathrm{a}, \mathrm{b}$ demonstrate silyl concentration, that is the main component, contributing to the film growth.
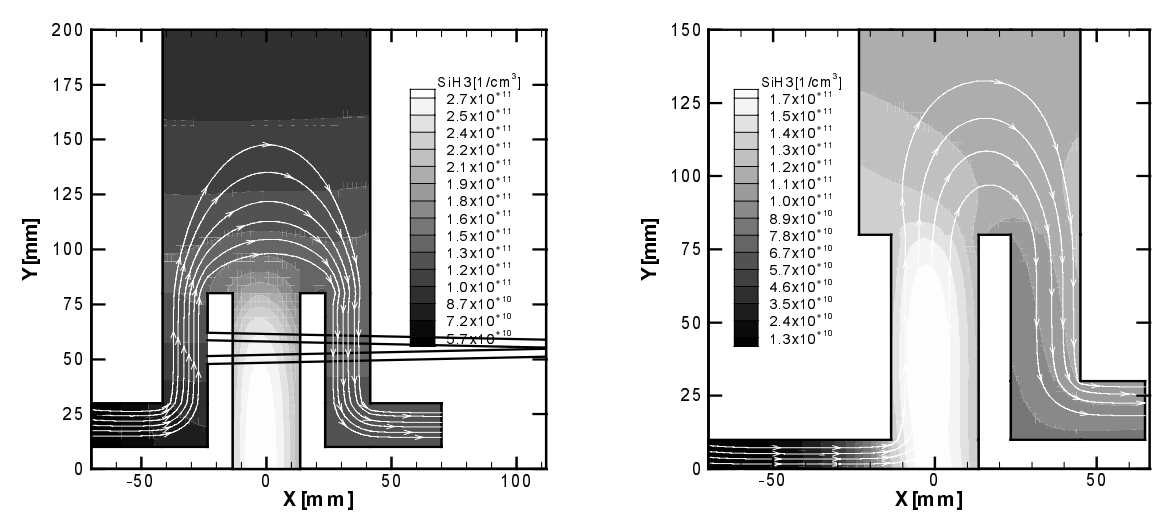

Fig. 8. Simulation results: stream lines and silyl concentration field. a , b - different geometrical configuration of reactors

On the next stage of our work, parallelization efficiency on a task-level was analyzed. Numerous tests showed that due to specificity of the problem, good results of parallel algorithm implementation for an individual task is possible only for a certain range of parameters, when communication overheads are minimal.

Figure 9 illustrates the typical performance result for 'unfavorable' task. Here speedup coefficient was calculated as the ratio of computing time with one processor to computing time for the number processors used. Analysis of communication traffic and workload balance showed, that freezing of speedup with number of processors is caused by too large time of data exchange comparatively with calculation time at every iteration. 
One possible way to improve this situation is to replace consecutive exchange between processors with more sophisticated algorithm configured as a processor tree.

Some experiments with different Myrinet communication environment drivers and versions of MPI showed that it is possible to achieve better performance (up to $30 \%$ ), but the tendency of freezing speedup coefficient still remains, especially for the middle-scale tasks, where number of beams is not large enough and the fact that their number is not divisible by total number of processors plays a significant role in load imbalance.

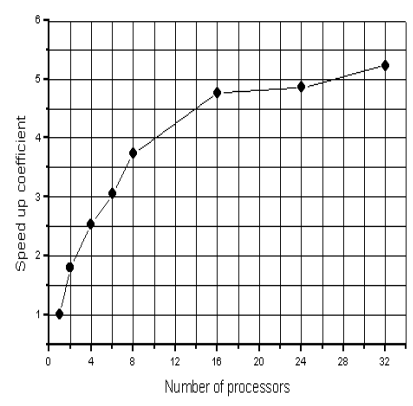

Fig. 9. Speed up coefficient

Besides, it is clear that the faster communications and the larger cash memory would help to overcome the difficulties, but we believe that some advantages also can be won by modification of the algorithms.

Working on the project, we discovered a new problem, when our simulation environment on the cluster was open for remote testing and many parallelized programs were submitted for execution on different number of processors. Serious processors workload imbalance was revealed. Attempts of using standard free-ware utilities, like queue, PBS, DQS, failed because algorithms used there do not suit the specific features of the program. For example, queue looks for the least loaded processor and sends a new process there. In the case of a large amount of data to be sent and heavy network traffic, this operation makes the target processor workload even less, and balance manager sends there one more task, collapsing whole calculation process. Under these circumstances, development (or using already produced) dynamic load balancing environment becomes the vital need on the way of creating general purpose efficient software for scientific community.

\section{Conclusion and Future Work}

Model and numerical algorithm for simulation of amorphous hydrogenated silicon films deposition were developed, MPI-based computing environment and advanced interactive software with graphic user interface, real-time visualization system and Web access were created, and large-scale parameter space investigations were successfully carried out on two Linux clusters.

Task-level parallelization algorithm using multi-block regular grid with beam distribution was applied for parallel implementation. It provided rather good parallelization efficiency for this class of CFD problem. However, it turned out that due to large communication overheads for most of the tasks considered, system performance almost stops speeding up with increasing of processors number, after a certain 'critical' number. The modification of parallelization algorithm is planned in order to eliminate possible bottlenecks, and some improvements will be made in 
initial beams distribution among processors for better load balancing. Possible ways of the communication environment modernization will be also investigated.

Considerable efforts should be made in development of dynamic load balancing environment in order to create a multi-scale multi-user multi-purpose parallel server software to provide an effective utilization of cluster computer power.

\section{References}

1. Yu.V. Lapin, M.Kh. Strelets. Internal flows of gas mixtures. Moscow, Nauka, 1989 (in Russian)

2. M.I. Zhilyaev, V.A. Schweigert, I.V. Schweigert. Simulation of RF silane discharge. Appl. Mech. Tech. Phys. V. 35, N 1, 1994, pp. 13-21

3. M.N. Kogan. Dynamics of rarefied gas. Moscow, Nauka, 1967 (in Russian)

4. Yu.E. Gorbachev, M.A. Zatevakhin, I.D. Kaganovich. Simulation of the growth of hydrogenated amorphous silicon films from an rf plasma. Tech. Phys. V. 41, N 12, 1996, pp. $1247-1258$

5. S.K. Godunov, V.S. Riabenkii. Difference schemes. Moscow, Nauka, 1973 (in Russian)

6. Yang J.Y., Hsu C.A. High-Resolution, Nonoscillatory Schemes for Unsteady Compressible Flows. AIAA J., V. 30, N 6, 1992, pp. 1570-1575

7. F. Casier, H. Deconinck, Ch. Hirsch. A class of bidiagonal schemes for solving the Euler Equations. AIAA J. V. 22, N 11, pp. 1556-1563

8. V.L. Varentsov, A.A. Ignatiev. Numerical investigations of internal supersonic jet targets formation for storage rings. Nuclear Instruments and Methods in Physics Research A 413, 1998, pp. 447-456

9. A.A.Ignatiev. Regular grid generation with mechanical approach. Mathematical Modelling, V. 12, N 2, 2000, pp. 101-105 (in Russian)

10. G.J. Nienhuis, W.J. Goedheer, E.A.G. Hamers, W.G.J.H.M. van Sark, and J. Bezemer, A self-consistent fluid model for $\mathrm{RF}$ dischargdes in $\mathrm{SiH}_{4}-\mathrm{H}_{2}$ compared to experiments. J. Appl. Phys. V.82, 1997, pp.2060-2071

11. Yu.E. Gorbachev, M.A. Zatevakhin, V.V. Krzhizhanovskaya, V.A. Schweigert. Special Features of the Growth of Hydrogenated Amorphous Silicon in PECVD reactors. Tech. Phys. V. 45 N 8, 2000, pp.1032-1041 